\title{
Fragmented QRS formation may be associated with increased carotid intima-media thickness in patients with end-stage renal
} disease

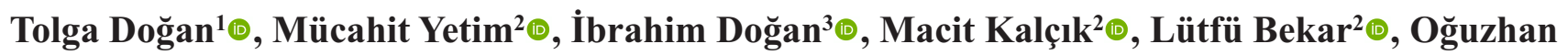 \\ Çelik ${ }^{4} \oplus$, Yusuf Karavelioğlu² $\odot$
}

${ }^{1}$ Department of Cardiology, Bursa Yüksek Ihtisas Training and Research Hospital, Bursa, Turkey

${ }^{2}$ Department of Cardiology, Hitit University, Çorum Training and Research Hospital, Çorum, Turkey

${ }^{3}$ Department of Nephrology, Hitit University, Çorum Training and Research Hospital, Çorum, Turkey

${ }^{4}$ Department of Cardiology, Muğla Sitkı Koçman University, Faculty of Medicine, Muğla, Turkey

\section{ABSTRACT}

Objectives: The aim of this study was to evaluate the relationship between carotid intima-media thickness and the presence of fragmented QRS in end-stage renal disease patients.

Methods: The study included 100 end-stage renal disease patients who received hemodialysis treatment. Two groups were formed according to the presence of electrocardiography and fragmented QRS: the fragmented QRS (+) (Group I) and the fragmented QRS (-) (Group II). Echocardiographic measurements of the cardiac dimensions and carotid intima-media thickness were examined.

Results: The study population included 41 end-stage renal disease patients with fragmented QRS (48.8\% male) and a control group of 59 end-stage renal disease patients without fragmented QRS (35.6\% male) on electrocardiography. Left ventricular end systolic diameters $(p=0.012)$,left ventricular end diastolic diameters $(p<0.001)$, left atrial diameter $(p=0.001)$, interventricular septal thickness $(p<0.001)$, posterior wall thickness $(p<0.001)$ and left ventricular hypertrophy $(p<0.001)$, blood urea nitrogen $(p=0.011)$, creatinine $(p=0.014)$, uric acid $(p<0.05)$ and parathyroid hormone $(p<0.05)$ values were found to be significantly increased in the fragmented QRS (+) group. The right and left carotid intima-media thickness values were significantly higher in fragmented QRS $(+)$ patients than fragmented QRS (-) patients (right:[0.81 \pm 0.19$]$ vs [0.62 \pm 0.14$] \mathrm{mm}, p$ $<0.001)$ and left: [0.83 \pm 0.19 vs $64 \pm 0.14], p<0.001$; respectively).

Conclusions: In end-stage renal disease patients, the presence of fragmented QRS, left ventricular hypertrophy and increased carotid intima-media thickness are important markers in the evaluation of the inflammatory process of atherosclerosis.

Keywords: fQRS, end-stage renal disease, carotid intima-media thickness, left ventricular hypertrophy

E nd-stage renal disease (ESRD) is an independent risk factor for cardiovascular (CV) complications such as sudden cardiac death, arrhythmias and heart failure. Impaired renal function causes increased in- flammation and coagulation, anemia, left ventricular hypertrophy, endothelial dysfunction, and arterial calcification $[1,2]$. ESRD patients have been reported to experience 2 -fold more $\mathrm{CV}$ events than the general 
population [3]. Carotid intima-media thickness (cIMT) is a non-invasive marker that is known to be associated with the presence of coronary artery disease and stroke $[4,5]$ and is an independent predictor of CV morbidity and mortality in ESRD patients. It has been reported that cIMT can strongly predict future $\mathrm{CV}$ events and mortality in chronic renal disease (CKD) patients [6]. Endothelial dysfunction and subsequent atherosclerosis are held responsible for increased intima thickness while increased media thickness results from smooth muscle hypertrophy due to hypertension. Carotid arteries are the most frequently used vessels because of the superficial location, ease of visualization, size and immobility [7].

Fragmented QRS (fQRS) complexes are a depolarization abnormality and fQRS is a marker of myocardial fibrosis or scar tissue $[8,9]$. It is shown that LV diastolic dysfunction in hemodialysis patients with fQRS is more severe than in patients without fQRS, and fQRS is associated with systolic and diastolic dysfunction of the left ventricle in kidney transplant patients. It was noted that fQRS can be used for risk classification in hemodialysis (HD) patients [10].

The aim of this study was to evaluate the relationship between cIMT and the presence of fQRS in ESRD patients.

\section{METHODS}

This study included 100 ESRD patients who had been receiving HD treatment three times a week for at least six months. Routine 12-lead ECGs were obtained in the patients who were then separated into two groups as the fQRS (+) group (Group I) and the fQRS
(-) group (Group II). cIMT measurements were taken of all the patients. Demographic characteristics, comorbidities and medication use were recorded and routine biochemical tests were performed. The study protocol was approved by the Erciyes University, Kayseri. Local Ethics Committee of the hospital in accordance with the Declaration of Helsinki and Good Clinical Practice Guidelines and written informed consent was obtained from all participants. Patients with acute coronary artery syndrome, rheumatic heart disease, cardiomyopathy, pregnancy, or atrial fibrillation were not included in the study. ECGs with pace rhythm, typical bundle branch block or any kind of significant conduction abnormality were also excluded from the study.

\section{Biochemical Assessment}

Blood samples were collected in the morning of a weekday from an antecubital vein after 12-hours of fasting without dialysis. Routine serum biomarkers were examined, including sodium $(\mathrm{Na})$, potassium $(\mathrm{K})$, calcium $(\mathrm{Ca})$, phosphorus, glucose, blood urea nitrogen $(\mathrm{BUN})$, creatinine $(\mathrm{Cr})$, uric acid, total cholesterol, low density lipoprotein cholesterol (LDL-C), high density lipoprotein cholesterol (HDL-C), triglyceride (TG), albumin, total protein, alkaline phosphatase, parathormone (PTH), ferritin, and high sensitive c-reactive protein (hsCRP). Values were calculated using standard laboratory methods (Beckmann Coulter aU5800 Autoanalyser, Beckmann Coulter Inc, Brea, California).

\section{Detection and Definition of fQRS}

Standard 12-lead electrocardiograms (ECGs) were obtained from all patients on a Nihon Kohden Cardio-

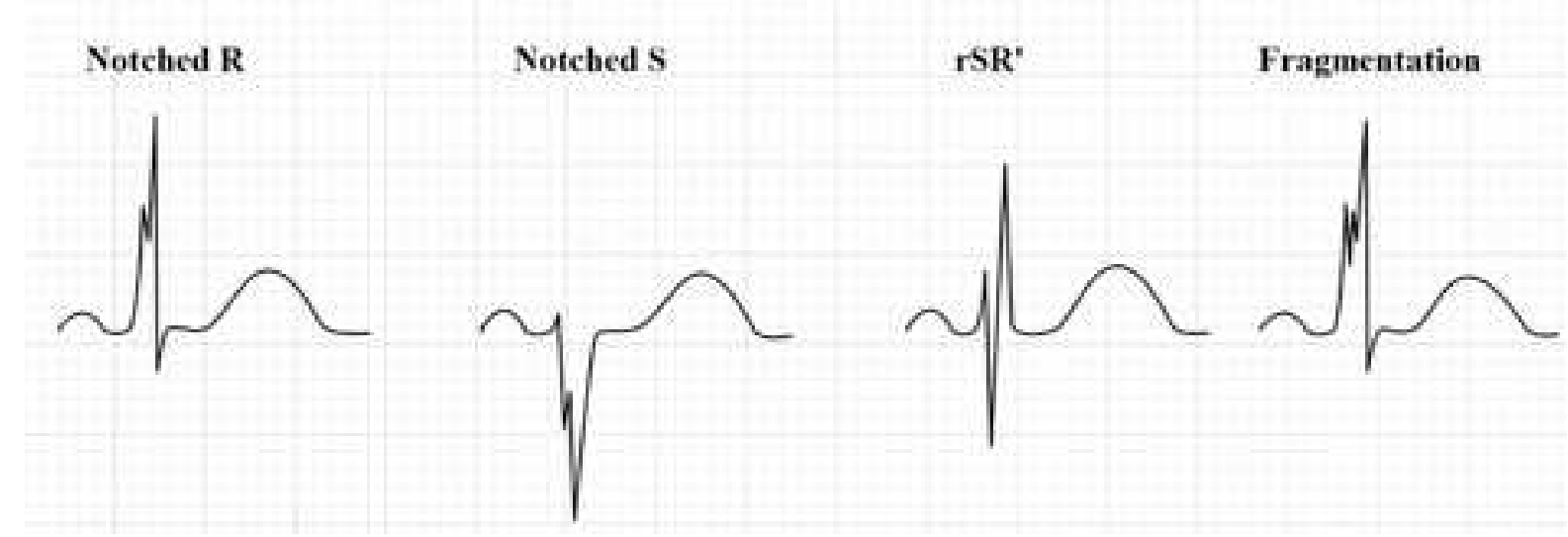

Fig. 1. Fragmented QRS examples. 
fax ECG-9132 device with a paper-speed of $25 \mathrm{~mm} / \mathrm{s}$, amplitude of $10 \mathrm{~mm} / \mathrm{mv}$, and a filter range of 0.15 to $100 \mathrm{~Hz}$. fQRS was defined as the presence of an additional $\mathrm{R}$ wave ( $\mathrm{R}$ '), notching of the $\mathrm{R}$ or $\mathrm{S}$ wave, or the presence of fragmentation (more than one R') in two contiguous leads (Fig. 1) [11]. Typical right or left bundle branch block pattern (QRS $\geq 120 \mathrm{~ms}$ ) and incomplete right bundle branch block were excluded. The ECGs were analyzed by 2 independent, experienced cardiologists, who were blinded to all data.

Transthoracic echocardiography was applied to all the patients by two cardiologists using a Vivid 5 echocardiography device (GE Vingmed Ultrasound AS, Horten, Norway), and $3.2 \mathrm{mHz}$ adult prob. Echocardiographic measurements of the cardiac dimensions were taken using parasternal short axis scanning with the patient in the left supine position, according to the American Society of Echocardiography guidelines [12]. Basic measurements included left ventricular end diastolic and end systolic diameters, left ventricular posterior wall thickness (PWT) interventricular septal thickness (IVST), end-diastolic and end-systolic ventricular diameters (LVEDD and LVESD) and left atrial diameter (LAD). Left ventricular ejection fraction (LVEF) was measured using the biplane Simpson and Teicholz method [13].

\section{Measurement of Carotid Intima-Media Thickness}

Carotid IMT measurements were taken with the patient in the supine position. Ultrasonography examinations were performed by two cardiologists. (Philips Ultrasound, Bothell, WA 98021 USA, L18-5 vascular probe). The morphology of both arterial carotid arteries, internal carotid artery and carotid bulb were examined in detail. The presence of atherosclerotic plaque and IMT were evaluated separately. Only the posterior (remote) wall was evaluated, and the IMT was measured in the area $1 \mathrm{~cm}$ proximal of the carotid bifurcation. Three different scanning angles were used: anterior oblique, lateral, and posterior oblique. The average value of the measurements obtained at these angles was calculated. For ultrasound analysis, the intima-media thickness was measured via the characteristic echogenicity of the lumen-intima and mediaadventitia surfaces.

\section{Statistical Analysis}

Data obtained in the study were analyzed statisti- cally using IBM SPSS Statistics for Windows, Version 19.0. software (IBM Corp. Armonk, NY, USA). Conformity of the variables to normal distribution was examined using analytical methods (Kolmogorov-Smirnov/Shapiro-Wilk test). Descriptive statistics were reported as mean \pm standard deviation (SD) values for continuous variables with normal distribution and as frequency (n) and percentage (\%) for categorical variables. Continuous variables were compared between groups using the Student's t-test when data distribution was normal. Comparisons of categorical variables were made using the $\chi^{2}$ test. A value of $p<0.05$ was accepted as statistically significant.

\section{RESULTS}

A total of 100 patients ( $59 \%$ female, median age $55.19 \pm 13$ years) were included in the study. The study population included 41 ESRD patients with fQRS (48.8\% male) on ECG and a control group of 59 ESRD patients without fQRS (35.6\% male). The demographic characteristics, medications and comorbidities of both groups are shown in Table 1. Age, gender, body mass index (BMI), duration of hemodialysis (the time since the first entry into hemodialysis), diabetes mellitus, hypertension, smoking status and medications were similar in the two groups. The laboratory test results and echocardiographic findings of the ESRD patients with and without fQRS are presented in Table 2. LVESD $(p=0.012)$, LVEDD $(p<0.001)$, LAD $(p=0.001)$, IVST $(p<0.001)$, PWT $(p<0.001)$ and left ventricular hypertrophy (LVH) $(p<0.001)$ were found to be significantly increased in the fQRS $(+)$ group (Table 2). Serum biomarkers of BUN $(p=$ $0.011)$, creatinine $(p=0.014)$, uric acid $(p<0.05)$ and PTH $(p<0.05)$ were found to be significantly increased in the fQRS $(+)$ group. The right and left cIMT values were significantly higher in fQRS $(+)$ patients than in fQRS (-) patients (right: [0.81 \pm 0.19$]$ vs [0.62 $\pm 0.14] \mathrm{mm}, p<0.001)$ and left: $[0.83 \pm 0.19$ vs $64 \pm$ $0.14], p<0.001$; respectively).

\section{DISCUSSION}

The results of this study demonstrated that the 
Table 1. The demographic characteristics, medications, laboratory tests and co-morbidities of the study population

\begin{tabular}{|c|c|c|c|}
\hline & $\begin{array}{c}\text { fQRS (+) Group } \\
(n=41)\end{array}$ & $\begin{array}{c}\text { fQRS (-) Group } \\
(n=59)\end{array}$ & $p$ value \\
\hline Age (years) & $56.5 \pm 12.3$ & $55.1 \pm 14.4$ & 0.632 \\
\hline Gender (M/F) & $20 / 21$ & $21 / 38$ & 0.187 \\
\hline $\operatorname{BMI}\left(\mathrm{kg} / \mathrm{m}^{2}\right)$ & $27.2 \pm 4.8$ & $25.9 \pm 4.6$ & 0.205 \\
\hline Smoker (n, \%) & 12.2 & 8.5 & 0.542 \\
\hline Diabetes Mellitus (\%) & 29.3 & 27.1 & 0.814 \\
\hline Hypertension (\%) & 80.5 & 72.9 & 0.381 \\
\hline Duration of hemodialysis (month) & $78.2 \pm 62.3$ & $65.6 \pm 52.3$ & 0.279 \\
\hline CAD $(\%)$ & 70.7 & 57.6 & 0.182 \\
\hline CVD $(\%)$ & 2.4 & 5.1 & 0.642 \\
\hline PAD (\%) & 9.8 & 11.9 & 0.740 \\
\hline BUN (g/dL) & $57.3 \pm 15.8$ & $49.2 \pm 15.3$ & 0.011 \\
\hline Creatinine $(\mathrm{g} / \mathrm{dL})$ & $7.4 \pm 2$ & $6.5 \pm 1.5$ & 0.014 \\
\hline GFR (mL/min) & $8 \pm 2.5$ & $8.3 \pm 1.9$ & 0.428 \\
\hline Uric Acid (mg/dL) & $5.2 \pm 1.5$ & $4.6 \pm 1.3$ & 0.041 \\
\hline $\mathrm{Ca}(\mathrm{mg} / \mathrm{dL})$ & $8.9 \pm 0.9$ & $9 \pm 0.7$ & 0.617 \\
\hline Phosphorus ( mg/dL ) & $4.6 \pm 1$ & $4.6 \pm 1.4$ & 0.795 \\
\hline PTH (pg/mL) & $510 \pm 467$ & $354 \pm 250$ & 0.033 \\
\hline Total Protein (gr/dL) & $7.2 \pm 0.6$ & $7.4 \pm 0.7$ & 0.254 \\
\hline Albumin (g/dL) & $4 \pm 0.3$ & $4.1 \pm 0.46$ & 0.550 \\
\hline Total Cholesterol (mg/dL) & $107 \pm 38$ & $189 \pm 46$ & 0.751 \\
\hline Glucose (mg/dL) & $107 \pm 38$ & $120 \pm 67$ & 0.256 \\
\hline $\mathrm{Hb}(\mathrm{gr} / \mathrm{dL})$ & $12.6 \pm 1.7$ & $12.6 \pm 1.5$ & 0.972 \\
\hline ACEi/ARB (\%) & 17.1 & 16.9 & 0.987 \\
\hline B Blockers (\%) & 46.3 & 50.8 & 0.658 \\
\hline $\mathrm{CCB}(\%)$ & 53.7 & 47.5 & 0.542 \\
\hline Statins $(\%)$ & 17.1 & 15.3 & 0.807 \\
\hline
\end{tabular}

$\mathrm{BMI}=$ Body Mass Index, CAD = Coronary Artery Disease, CVD = Cerebrovascular Disease, PAD = Peripheral Artery Disease, BUN = Blood Urea Nitrogen, GFR = Glomerular Filtration Rate, PTH = Parathormone, ACEi/ARB $=$ Angiotensinconverting Enzyme Inhibitors/Angiotensin Receptor Blockers, BB = Beta Blockers, CCB = Calcium Channel Blockers

cIMT values were significantly higher in the fQRS $(+)$ ESRD group than in the fQRS(-) ESRD group. In addition, LVESD, LVEDD, LAD, IVST, PWT diameters, BUN, creatinine and PHT levels were higher in fQRS (+) ESRD patients.

Cardiovascular diseases are still the most common cause of death for ESRD patients [14, 15].The underlying pathophysiological mechanism is atherosclerosis [16]. Therefore, CV risk assessment is very important and vascular imaging is one of the risk assessment methods for atherosclerosis [17]. Carotid artery doppler ultrasound is an inexpensive and non-invasive imaging method. Increased cIMT reflects subclinical atherosclerosis. Various $\mathrm{CV}$ guidelines recommend the use of this measurement for the evaluation of target organ damage $[18,19]$. Nonetheless, the lack of a standardized cIMT value or consistent measurement methods for increased IMT limits the use of this parameter 
Table 2. The cIMT and echocardiographic findings of both study groups

\begin{tabular}{lccc}
\hline & $\begin{array}{c}\text { fQRS (+) Group } \\
(\mathbf{n = 4 1 )}\end{array}$ & $\begin{array}{c}\text { fQRS (-) Group } \\
(\mathbf{n = 5 9 )}\end{array}$ & $\boldsymbol{p}$ value \\
\hline EF $(\%)$ & $59.5 \pm 7.7$ & $60 \pm 7.2$ & 0.735 \\
LVESD $(\mathrm{mm})$ & $33.7 \pm 6.6$ & $30.6 \pm 5.4$ & $\mathbf{0 . 0 1 2}$ \\
LVEDD $(\mathrm{mm})$ & $50.8 \pm 6.3$ & $45.9 \pm 5.2$ & $<\mathbf{0 . 0 0 1}$ \\
IVS $(\mathrm{mm})$ & $13.2 \pm 1.6$ & $10.7 \pm 1.6$ & $<\mathbf{0 . 0 0 1}$ \\
PW $(\mathrm{mm})$ & $12.8 \pm 1.4$ & $10.5 \pm 1.4$ & $<\mathbf{0 . 0 0 1}$ \\
LAD $(\mathrm{mm})$ & $38.2 \pm 4.7$ & $34.6 \pm 5.4$ & $\mathbf{0 . 0 0 1}$ \\
LVH $(\%)$ & 94.9 & 42.4 & $<\mathbf{0 . 0 0 1}$ \\
Mean right cIMT $(\mathrm{mm})$ & $0.81 \pm 0.19$ & $0.62 \pm 0.14$ & $<\mathbf{0 . 0 0 1}$ \\
Mean left cIMT $(\mathrm{mm})$ & $0.83 \pm 0.19$ & $0.64 \pm 0.14$ & $<\mathbf{0 . 0 0 1}$ \\
\hline
\end{tabular}

$\mathrm{EF}=$ ejection fraction, $\mathrm{LVESD}=$ left venticle end systolic diameter, $\mathrm{LVEDD}=$ left venticle end diastolic diameter, IVS $=$ Interventricular septal diameter, $\mathrm{PW}=$ Posterior wall diameter, $\mathrm{LAD}=$ left atrial diameter, $\mathrm{LVH}=$ left ventricular hypertrophy, cIMT $=$ Carotis intima-media diameter

in clinical trials [20]. Therefore, it may need confirmation with other prognostic markers.

In a study by Benedeto et al. [21], cIMT was associated with LV concentric hypertrophy in dialysis patients and was reported to be an independent predictor of CV death, which could be useful for risk classification in the dialysis population. In the current study, a similar relationship between concentric hypertrophy of LV and increased cIMT was found. LVH is a marker of target organ damage, which can be measured non-invasively using echocardiography, and is associated with CV morbidity and mortality in HD patients [22]. In end-organ damage such as LVH, hypertrophy occurs in myocytes due to collagen deposition in interstitial tissue. In this process, myocardial fibrosis may occur in ESRD patients in the presence of $\mathrm{LVH}$ and due to progressive increase in renal dyfunction [23].

Myocardial fibrosis occurs as a result of excessive deposition of type 1 collagen fibers into the interstitium, around intramyocardial arteries and arterioles. CKD is one of the reasons for the exaggerated synthesis of collagen by cardiac fibroblasts and myofibroblasts. Exposure to oxidative stress, inflammation and hemodynamic overload starting from the early stages of the CKD and also increased release of cytokines such as cardiotrophin-1 and transforming growth factor- $\beta 1$ or TGF- $\beta 1$ contribute to myocardial scar formation. In the more advanced stages of CKD, uremic toxins, anemia, hyperphosphatemia, parathormone excess, and vitamin D deficiency may also cause cardiovascular damage and increased myocardial scarring $[24,25]$. Mechanical overload due to LVH and dias- tolic dysfunction in CKD also leads to exaggerated collagen deposition [26].

fQRS formation reflecting delayed ventricular conduction is a non-invasive parameter used to evaluate atherosclerosis. It is also associated with fibrosis, myocardial scar and ischemia [8]. Several studies have proven the utility of fQRS in predicting major adverse cardiac events (MACE) and all-cause mortality in patients with coronary artery disease $[27,28]$. In addition, LV deformation and increased LVH incidence have been reported in hypertensive patients with fQRS. Although there are limited studies on fQRS in patients with ESRD, it has been reported that fQRS may be useful in determining LV dysfunction in renal transplant patients. It has also been shown to be independently associated with normal LVEF and subclinical LV dysfunction in ESRD patients and aortic stiffness has been found to be an important predictor in hemodialysis patients $[29,30]$. There is a known association between hypertensive patients with fQRS and impaired diastolic functions [31]. In the current study, increased LVESD, LVEDD, LAD, IVST and PWT parameters in fQRS (+) ESRD patients were associated with impaired diastolic function secondary to LVH and this may lead to myocardial fibrosis. Myocardial scar formation and diastolic dysfunction due to $\mathrm{LVH}$, the presence of fQRS in ECG as well as increased cIMT thickness could demonstrate that ESRD patients may be more prone to atherosclerosis.

Increased BUN, creatinine, and PTH levels reflect impaired kidney function. High values of these parameters in fQRS $(+)$ patients may support the acceleration of the atherosclerotic process. 


\section{Limitations}

Limitations of this study were primarily the nonrandomized, single centered nature and limited number of patients. Due to the lack of a standardized cIMT value, these results may differ from those of largescale studies. There is a clear need for further more extensive studies of the traditional risk factors and high-risk populations. Unfortunately, due to the nonanalytical design of our study we were not able to invastigate rigid outcomes in our research. Nevertheless, our study may be helpful during designing hypotheses for advanced trials.

\section{CONCLUSION}

In ESRD patients, the presence of fQRS, LVH and increased CIMT are important markers in the evaluation of the inflammatory process of atherosclerosis. Increased cIMT is related with fQRS presence in ESRD patients.

\section{Authors' Contribution}

Study Conception: TD; Study Design: MY; Supervision: TD; Funding: TD; Materials: ID; Data Collection and/or Processing: TD; Statistical Analysis and/or Data Interpretation: OÇ; Literature Review: MK; Manuscript Preparation: LB and Critical Review: YK.

\section{Conflict of interest}

The authors disclosed no conflict of interest during the preparation or publication of this manuscript.

\section{Financing}

The authors disclosed that they did not receive any grant during conduction or writing of this study.

\section{REFERENCES}

1. Go AS, Chertow GM, Fan D, McCulloch CE, Hsu CY. Chronic kidney disease and the risks of death, cardiovascular events, and hospitalization. N Engl J Med 2004;351:1296-305.

2. Turner S, Onalan O, Bickle B. Prevention of de ath in chronic kidney disease: the role of implantable cardioverter defibrillators. CANNT J 2009;19:29-36.

3. Collins AJ, Li S, Gilbertson DT, Chen SC, Herzog CA. Chronic kidney disease and cardiovascular disease in the Medicare population. Kidney Int Suppl 2003;87:S24-31.
4. Liviakis L, Pogue B, Paramsothy P, Bourne A, Gill EA. Carotid intima-media thickness for the practicing lipidologist. J Clin Lipidol 2010;4:24-35.

5. O’Leary DH, Polak JF, Kronmal RA, Manolio TA, Burke GL, Wolfson SK, Jr. Carotid-artery intima and media thickness as a risk factor for myocardial infarction and stroke in older adults. Cardiovascular Health Study Collaborative Research Group. N Engl J Med 1999;340:14-22.

6. Roumeliotis A, Roumeliotis S, Panagoutsos S, Theodoridis M, Argyriou C, Tavridou A, et al. Carotid intima-media thickness is an independent predictor of all-cause mortality and cardiovascular morbidity in patients with diabetes mellitus type 2 and chronic kidney disease. Ren Fail 2019;41:131-8.

7. Wong M, Edelstein J, Wollman J, Bond MG. Ultrasonic-pathological comparison of the human arterial wall: verification of intima-media thickness. Arterioscler Thromb 1993;13:482-6.

8. Basaran Y, Tigen K, Karaahmet T, İsiklar I, Cevik C, Gurel E, et al. Fragmented QRS complexes are associated with cardiac fibrosis and significant intraventricular systolic dyssynchrony in nonischemic dilated cardiomyopathy patients with a narrow QRS interval. Echocardiography 2011;28:62-8.

9. Park SJ, On YK, Kim JS, Park SW, Yang JH, Jun TG, et al. Relation of fragmented QRS complex to right ventricular fibrosis detected by late gadolinium enhancement cardiac magnetic resonance in adults with repaired tetralogy of Fallot. Am J Cardiol 2012;109:110-5.

10. Güçlü A, Nar G, İçli A, Özhan N, Sezer S. Relationship between fragmented QRS complex and aortic stiffness in chronic hemodialysis patients. Med Princ Pract 2017;26:66-70.

11. Pietrasik G, Zaręba W. QRS fragmentation: diagnostic and prognostic significance. Cardiol J 2012;19:114-21.

12. Sahn DJ, DeMaria A, Kisslo J, Weyman A. Recommendations regarding quantitation in M-mode echocardiography: results of a survey of echocardiographic measurements. Circulation 1978;58:1072-83.

13. Wood PW, Choy JB, Nanda NC, Becher H. Left ventricular ejection fraction and volumes: it depends on the imaging method. Echocardiography 2014;31:87-100.

14. Fortes PC, Versari PH, Stinghen AE, Pecoits-Filho R. Controlling inflammation in peritoneal dialysis: the role of pd-related factors as potential intervention targets. Perit Dial Int 2007;27 Suppl 2:S76-81.

15. Bakris GL, Agarwal R, Anker SD, Pitt B, Ruilope LM, Nowack C, et al. Design and baseline characteristics of the finerenone in reducing kidney failure and disease progression in diabetic kidney disease trial. Am J Nephrol 2019;50:333-44.

16. Maraj M, Kuśnierz-Cabala B, Dumnicka P, Gala-Bladzinska A, Gawlik K, Pawlica-Gosiewska D, et al. Malnutrition, Inflammation, Atherosclerosis Syndrome (MIA) and Diet Recommendations among End-Stage Renal Disease Patients Treated with Maintenance Hemodialysis. Nutrients 2018;10:69.

17. Carpenter M, Sinclair H, Kunadian V. Carotid intima media thickness and its utility as a predictor of cardiovascular disease: a review of evidence. Cardiol Rev 2016;24:70-5.

18. Graham I, Atar D, Borch-Johnsen K, Boysen G, Burell G, Cifkova R, et al. European guidelines on cardiovascular disease prevention in clinical practice: executive summary. Eur J Cardio- 
vasc Prev Rehabil 2007;14 Suppl 2:E1-40.

19. Mancia G, De Backer G, Dominiczak A, Cifkova R, Fagard R, Germano G, et al. 2007 Guidelines for the management of arterial hypertension: The Task Force for the Management of Arterial Hypertension of the European Society of Hypertension (ESH) and of the European Society of Cardiology (ESC). Eur Heart J 2007;28:1462-536.

20. Ravani A, Werba JP, Frigerio B, Sansaro D, Amato M, Tremoli E, et al. Assessment and relevance of carotid intimamedia thickness (C-IMT) in primary and secondary cardiovascular prevention. Curr Pharm Des 2015;21:1164-71.

21. Benedetto FA, Mallamaci F, Tripepi G, Zoccali C. Prognostic value of ultrasonographic measurement of carotid intima media thickness in dialysis patients. J Am Soc Nephrol 2004;12:245864.

22. Selim G, Stojceva-Taneva O, Tozija L, Zafirova-Ivanovska B, Spasovki G, Gerasimovska V, et al. Uric acid and left ventricular hypertrophy: another relationship in hemodialysis patients. Clin Kidney J 2019;14:578-85.

23. Lopez B, Gonzalez A, Hermida N, Laviades C, Diez J. Myocardial fibrosis in chronic kidney disease: potential benefits of torasemide. Kidney Int Suppl 2008:111:S19-23.

24. Díez J. Mechanisms of cardiac fibrosis in hypertension. J Clin Hypertens (Greenwich). 2007;9:546-50.

25. Cozzolino M, Mangano M, Stucchi A, Ciceri P, Conte F,
Galassi A. Cardiovascular disease in dialysis patients. Nephrol Dial Transplant 2018;33(suppl 3):iii28-iii34.

26. Díez J, González A, López B, Querejeta R. Mechanisms of disease: pathologic structural remodeling is more than adaptive hypertrophy in hypertensive heart disease. Nat Clin Pract Cardiovasc Med 2005;2:209-16.

27. Ari H, Cetinkaya S, Ari S, Koca V, Bozat T. The prognostic significance of a fragmented QRS complex after primary percutaneous coronary intervention. Heart Vessels 2012;27:20-8.

28. Pietrasik G, Goldenberg I, Zdzienicka J, Moss AJ, Zareba W. Prognostic significance of fragmented QRS complex for predicting the risk of recurrent cardiac eventsin patients with Q-wave myocardial infarction. Am J Cardiol 2007;100:583-6.

29. Ulusoy S, Ozkan G, Adar A, Bektaş H, Kırış A, Celik S. Relationship between fragmented QRS complex and left ventricular systolic and diastolic function in kidney transplant patients. Prog Transplant 2014 24:146-51.

30. Adar A, Kiriş A, Ulusoy S, Ozkan K, Bektaş H, Okutucu S, et al. Fragmented QRS is associated with subclinical left ventricular dysfunction in patients with chronic kidney disease. Acta Cardiol 2014;69:385-90.

31. Kad1 H, Demir AK, Ceyhan K, Damar İH, Karaman K, Zorlu Ç. Association of fragmented QRS complexes on ECG with left ventricular diastolic function in hypertensive patients. Turk Kardiyol Dern Ars 2015;43:149-56. 